\title{
Genotype $\times$ Environment Interaction for Milk Production in Guernsey Cattle
}

\author{
W. F. Fikse*, R. Rekaya† and K. A. Weigelł \\ *Interbull Centre, Department of Animal Breeding and Genetics, \\ Swedish University of Agricultural Sciences, \\ S-750 07, Uppsala, Sweden \\ †Department of Animal and Dairy Science, \\ University of Georgia, Athens 30602 \\ ‡Department of Dairy Science, \\ University of Wisconsin, Madison 53706
}

\begin{abstract}
International genetic evaluations that use national genetic evaluation results as input need to acknowledge country boundaries. The current model for international evaluation treats each country as a genetically separate trait, i.e., assumes milk production to be similar within country, but different between countries. The use of cow performance records does not require such restriction, and allows for other statistical models to consider genotype $\times$ environment interaction. First-lactation records from 40,000 Guernsey cows in four countries (Australia, Canada, United States, and South Africa) were used to detect and describe genotype $\times$ environment interaction for milk production traits. Five statistical models were considered: single-trait acrosscounty (ST), single-trait across-country with heterogeneous residual variance (SThet), multiple-trait acrosscountry (MT), multiple-trait herd cluster model (HC), and reaction norm model (RR). For the herd cluster model, herds were clustered into groups based on information on herd management, genetic composition, and climate. Reaction norms describe the phenotype expressed by a genotype as a function of the environment, and was modeled by random regression on the herd average for peak milk yield as the descriptor of production environment. Gibbs sampling was used to make inferences about the parameters of interest, and models were compared based on goodness of fit and deviance information criterion. Posterior mode of the heritability for the single-trait model was 0.32 , and ranged from 0.15 to 0.53 for models SThet and MT. Posterior mode of the genetic correlations between countries estimated with model MT were generally high (0.78 to 0.90$)$. However, posterior SD were high (up to 0.15 for AustraliaSouth Africa), and values near unity for the genetic
\end{abstract}

Received March 27, 2002.

Accepted August 12, 2002

Corresponding author: F. Fikse; e-mail:Freddy.Fikse@hgen.slu.se. correlations were not unlikely. Model HC gave more precise inferences but lower goodness of fit compared with model MT. Results from model RR provided evidence for heterogeneity of genetic variances. This model was least supported by the data, probably because heterogeneity of residual variances was not considered. Among the models in this study, the one with homogeneous genetic and heterogeneous residual variances across countries fitted best to the data, and we expect a model for which the assumption of homogeneous genetic variance is relaxed to show an even better fit to the data.

(Key words: international genetic evaluation, Guernsey, lactation records)

Abbreviation key: DIC = deviance information criterion; HC = multiple-trait herd cluster model; $\mathbf{S T}=$ single-trait model; SThet $=$ single-trait model with heterogeneous residual variance across countries; $\mathbf{M T}=$ multiple-trait across country model; $\mathbf{R R}=$ reaction norm model (random regression).

\section{INTRODUCTION}

Utilization of preprocessed information for international genetic evaluation of dairy cattle puts several restrictions on the method of analysis. For example, if national breeding values for sires are used as input, then national genetic evaluation unit should be included in the statistical model. However, performance in neighboring countries with similar production environments cannot be treated as genetically identical traits, and possible genotype $\times$ environment interaction variation within country cannot be utilized. In addition, genetic correlations between countries do not only depend on genotype $\times$ environment interaction, but also on differences between national evaluation systems.

Access to individual performance records allows for international evaluations that can avoid some shortcomings of the use of national genetic evaluation results as input. Two procedures that consider variation in 
production environments between and within countries, and allow for more uniform processing of data, have recently been suggested for international genetic evaluation using performance records. The multipletrait herd cluster model (Weigel and Rekaya, 2000) stratifies herds according to production environment based on information on environmental descriptors. Production in each herd cluster is treated as a genetically distinct trait in the genetic evaluation model. Reaction norms (Kolmodin et al., 2002), on the other hand, describes the phenotype as a function of environmental descriptors. The function that relates phenotype to environment is unique to each genotype. Hence, the response to changes in environments can vary from one genotype to the other.

Several reports have been presented on investigations of the feasibility of joint, multinational genetic evaluations. For example, a Nordic cooperation has been established with the aim of developing a joint evaluation of dairy cattle in four Nordic countries (Pedersen et al., 2001). A single-trait across-country model was adopted, but a multiple-trait across-country model was also investigated. A multiple-trait across-country model was used for a joint evaluation of milking speed records from Germany and Austria (Sprengel et al., 2001). Breeding values for only one trait (country) were published, though, since the genetic correlations between traits (countries) were high. The majority of studies on multinational genetic evaluations either assumed a single-trait across-country model or treated performance in different countries as separate, though correlated traits. However, little consideration has been given as to whether country boundaries appropriately defined production environment, and whether genotype $\times$ environment interaction was optimally accounted for.

Numerous models for international genetic evaluations that use performance records can be contemplated, however, emphasis on identifying the order of models with respect to their ability to make correct inferences has thus far been limited. The objective of this study was to estimate genetic parameters for the multiple-trait herd cluster model, the reaction norm model, and three models that treat country as production environment, using data on Guernsey cows from four countries. These five models were compared with regard to their goodness of fit and model complexity.

\section{MATERIALS AND METHODS}

\section{Data}

First-lactation records on milk yield for 41,551 Guernsey cows produced between January 1990 and December 1999 from four countries were used, Australia (AUS), Canada (CAN), United States (USA), and
Table 1. Number, mean $(\mathrm{kg})$, and SD $(\mathrm{kg})$ of lactation records per country.

\begin{tabular}{lcll}
\hline & Number & Mean & SD \\
\hline Australia & 4803 & 4355 & 1195 \\
Canada & 3975 & 5532 & 1170 \\
United States & 32068 & 5854 & 1236 \\
South Africa & 705 & 4417 & 1154 \\
\hline
\end{tabular}

South Africa (ZAF). The original data files received from the four countries contained test-day yields and information on herd, region, date of calving, date of birth, sire, dam, maternal grandsire and maternal granddam. The data edits were applied and the procedure used for extension of incomplete lactations were described in detail by Fikse et al. (2003). Briefly, the original data were edited for minimum number of tests, minimum and maximum test-day yield and age at calving. Incomplete lactation records were extended to 305-d lactation length using Wood's (1967) function. A summary of the data used in the present study is in Table 1 . The lowest average milk production was realized in the countries practicing grazing systems. Standard deviation of 305$\mathrm{d}$ milk production was very similar for all four countries $(\sim 1200 \mathrm{~kg})$.

Pedigree information was completed with records for bulls extracted from the February 2000 routine international evaluation of Interbull for the Guernsey breed. In total, 611 bulls with daughters were identified, of which 98, 75, 498, and 33 had daughters with records in Australia, Canada, United States and South Africa, respectively.

\section{Statistical Models}

The comparison involved five models that differed in the way genotype $\times$ environment interaction was considered. The same three fixed effects were included in all five models, and these were herd-year-season of calving, heterosis class, and age at calving. Contemporary groups were made by clustering calvings within a herd based on the number of days between two consecutive calvings (Smith et al., 1991). Minimum contemporary group size was five, and the length of seasons was at most 7 mo. Breed designation codes of parents and grandparents were used to form heterosis classes, and resulted in four classes for animals with 100, 75, 50, and $25 \%$ of Guernsey genes, respectively.

Model ST. The simplest model in this study was a single-trait sire model, with one sire variance and one residual variance common to the whole population. Also one set of solutions for levels of age at calving and heterosis class was estimated for the whole population:

$$
y=\text { fixed }+ \text { sire }+e,
$$


where $y$ is the vector of lactation yields, fixed represents the vector of fixed effects for contemporary group, age at calving and heterosis class, sire is the vector of random sire effects, and e is the vector of residual effects.

Model SThet. Lactation yields were analyzed with a model that in addition to a sire effect contained countryspecific residual variances and fixed effects:

$$
\mathrm{y}_{\mathrm{i}}=\text { fixed }_{\mathrm{i}}+\text { sire }+\mathrm{e}_{\mathrm{i}}
$$

where $y_{i}$ is the vector of lactation yields in country $i(i$ $=1 \ldots 4)$, fixed $_{i}$ is the vector of fixed effect nested within country $i$, and $e_{i}$ is the vector of residual effects for country i. Thus, for each country a separate set of estimates for the residual variance and for levels of age at calving and heterosis class was computed. One sire variance common to all countries was assumed, which means that a unity genetic correlation among countries was assumed.

Model MT. Performance records from different countries were treated as genetically different traits in a conventional multiple-trait model. Genetic and environmental parameters were estimated specific to each country:

$$
\mathrm{y}_{\mathrm{i}}=\text { fixed }_{\mathrm{i}}+\operatorname{sire}_{\mathrm{i}}+\mathrm{e}_{\mathrm{i}}
$$

where sire $_{i}$ is the vector of random sire effects for country i. The sire effects were assumed to be correlated among countries, but residuals were uncorrelated since each daughter produced in one country only.

Model HC. Herds were clustered according to production environment, and lactation yields were subsequently analyzed with a multiple-trait sire model in which production in each cluster was treated as a separate trait:

$$
y_{j}=\text { fixed }_{j}+\operatorname{sire}_{j}+e_{j},
$$

where $y_{j}$ is the vector of lactation yields in cluster $j(j$ $=1 \ldots \mathrm{C}$ ), fixed $_{\mathrm{j}}$ denotes the vector of fixed effects nested within cluster $\mathrm{j}$, sire $\mathrm{j}_{\mathrm{j}}$ is the vector of random sire effects for cluster $j$, and $e_{j}$ is the vector of residual effects for cluster $\mathrm{j}$. This model yielded genetic parameters and fixed effects solutions specific to production environment rather than country. Herds were grouped into clusters with the K-means algorithm that minimized the within-cluster sums of squares (NAG, 2000). The distance was computed as the weighted squared difference for nine environmental descriptors. These environmental descriptors were found to be able to distinguish between production environments in the study by Fikse et al. (2003) on the same data. The weight given to each variable was based on both the genetic correlation between milk production in extreme environments ( 5 and $95 \%$ percentile values for herd averages of environmental descriptor) and the phenotypic correlation between herd averages for all environmental descriptors (Kinghorn, 1998). The values for genetic and phenotypic correlations were taken from the study by Fikse et al. (2003).

Model RR. Reaction norms for sires were estimated as random regressions of lactation yields on an environmental descriptor:

$$
\mathrm{y}=\text { fixed }+\operatorname{sire}(\mathrm{env})+\mathrm{e},
$$

where sire(env) is a random regression on env, the herd average milk peak yield. The random regression included an intercept and linear term that were treated as genetically correlated traits. One set of estimates for fixed effects was computed for the whole population. Herd average for milk peak yield was chosen as environmental descriptor, since genotype $\times$ environment interaction was most pronounced when this variable was used as environmental descriptor in the study by Fikse et al. (2003). They compared 15 environmental descriptors for their potential to reveal genotype $\times$ environment interaction, based on the genetic correlation between milk production in "poor" and "good" environment according to each descriptor.

\section{Implementation}

A Bayesian approach via the Gibbs sampler was implemented to obtain posterior distributions of the model parameters. Bounded uniform priors were used for the fixed effects. For sire breeding values a multivariate normal prior distribution was used. The prior distributions for the residual variances for all five models and the sire variance in models ST and SThet were bounded uniform distributions. For the sire covariance matrix a conjugate prior with two degrees of belief was used leading to weakly informative prior information. A single chain with a length of 150,000 was run for all five models. Burn-in and thinning parameters were determined for each chain separately with the Gibanal program (VanKaam, 1998).

\section{Model Comparison}

Support for each model was determined with the Bayesian Deviance Information Criterion (DIC). This criterion combines both goodness of fit and model complexity. It was computed as:

$$
D I C=\bar{D}+p_{D},
$$


Table 2. Mode and SD (in subscript) of the posterior distribution of the heritability for the models ST, SThet and MT. ${ }^{1}$

\begin{tabular}{|c|c|c|c|c|}
\hline Model & Australia & Canada & $\begin{array}{l}\text { United } \\
\text { States }\end{array}$ & $\begin{array}{l}\text { South } \\
\text { Africa }\end{array}$ \\
\hline ST & & 0. & 032 & \\
\hline SThet & $\begin{array}{lll}0.53 & 0.048\end{array}$ & $\begin{array}{lll}0.33 \quad 0.031\end{array}$ & $\begin{array}{lll}0.30 & 0.028\end{array}$ & $\begin{array}{lll}0.47 & 0.049\end{array}$ \\
\hline MT & $\begin{array}{lll}0.41 & 0.081\end{array}$ & $\begin{array}{lll}0.31 & 0.072\end{array}$ & $\begin{array}{lll}0.32 & 0.033\end{array}$ & $0.15 \quad 0.118$ \\
\hline
\end{tabular}

${ }^{1} \mathrm{ST}=$ single-trait model; SThet = single-trait model with heterogeneous residual variance across countries; $\mathrm{MT}=$ multiple-trait acrosscountry model.

where $\bar{D}$ is the posterior expectation of the Bayesian deviance. The Bayesian deviance is computed as:

$$
D(\bar{\theta})=-2 \log p(y \mid \theta) .
$$

$\mathrm{p}_{\mathrm{D}}$ is the effective number of parameters,

$$
p_{D}=\bar{D}-D(\bar{\theta})
$$

where $\bar{\theta}$ is the posterior mean of parameters in the model. For computational details see Spiegelhalter et al. (1998).

\section{RESULTS AND DISCUSSION}

\section{ST, SThet, and MT}

Posterior modes of the heritabilities ranged from 0.30 to 0.33 for CAN and USA for the ST, SThet and MT analyses, but varied more between models for AUS and $\mathrm{ZAF}$ (Table 2). The variation in estimates of variance components and ratios seemed largely determined by which model parameters were constrained. There were no constraints in model MT, and point estimates of variance components agreed well with preliminary within-country analyses (results not shown). For model SThet, however, a sire variance common to all countries was assumed, and the estimate seemed to be dominated by the USA data (Table 3). The posterior model of resid-

Table 3. Mode and SD (in subscript) of the posterior distribution of the sire and residual variance $\left(\times 1000 \mathrm{~kg}^{2}\right)$ for the models ST, SThet, and $\mathrm{MT}^{1}$

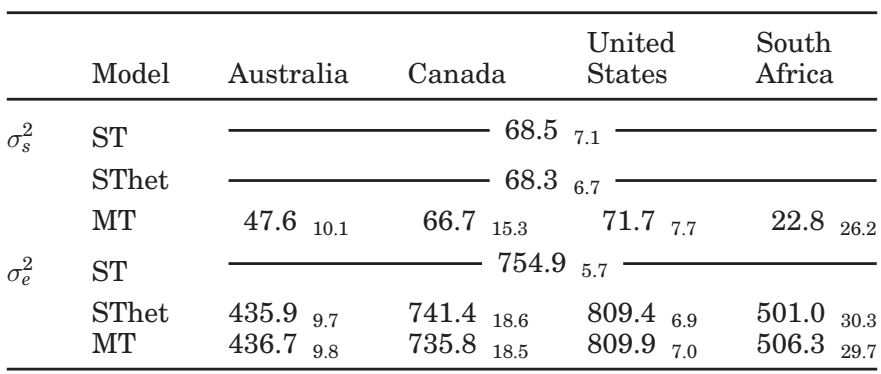

\footnotetext{
${ }^{1} \mathrm{ST}=$ single-trait model; SThet = single-trait model with heterogeneous residual variance across countries; $\mathrm{MT}=$ multiple-trait acrosscountry model.
}

Table 4. Modes and SD (in subscript) of the posterior distribution of the genetic correlations between countries for the multiple-trait across country model.

\begin{tabular}{llllll}
\hline & Canada & United States & \multicolumn{2}{l}{ South Africa } \\
\hline Australia & \multirow{2}{*}{0.900 .056} & 0.870 .064 & 0.87 & 0.149 \\
Canada & & 0.87 & 0.064 & 0.78 & 0.104 \\
United States & & & & 0.86 & 0.137 \\
\hline
\end{tabular}

ual variances were nearly the same for SThet and MT, and, as a consequence, heritabilities were higher for AUS and ZAF in model SThet compared to model MT. The heritabilities for AUS and ZAF in model SThet were higher than in ST because country-specific residual variances were assumed, and estimates of these parameters were lower for AUS and ZAF compared with the common residual variance in model ST.

Posterior SD of the variance components were highest for model MT (Tables 2 and 3). The uncertainty about the heritability for USA was only slightly different depending on model, but for the other three countries relaxing the assumption of a sire variance common to all countries more than doubled the posterior SD of heritabilities (Table 2). This exemplifies that the structure of the data was unbalanced and that the USA contributed the most information.

Posterior mode of genetic correlations between countries obtained with the MT model were between 0.86 and 0.90, except for the correlation between CAN and ZAF (Table 4). Genetic correlations with ZAF were estimated with much uncertainty, mainly due to the small amount of data in combination with the low number of bulls with daughters in both ZAF and one of the other three countries. Genetic correlations that involved AUS were higher in this study compared to correlations calculated from national evaluations for sires that are used by Interbull. A similar observation was made by Weigel et al. (2001) who used performance records of Holstein cows from 17 countries. The estimated genetic correlations may have been higher because the data used in this study spanned a shorter time period than did the data used by Interbull, and statistical models and number of lactations considered were different from national evaluations.

The contemporary group effects accounted for a proportionally larger part of the variation in observations in AUS and ZAF than in CAN and USA. The SD of observations was similar across countries (Table 1), however, the phenotypic variance computed as the sum of the genetic and residual variance differed considerably between countries [483, 792, 885 and $528(\times 1000$ $\left.\mathrm{kg}^{2}\right)$ in AUS, CAN, USA and ZAF, respectively]. In AUS, low input grazing systems are common practice, and production level is especially sensitive to variation in 
Table 5. Number of herds and proportion of a country's herds assigned to each cluster, and means of descriptive herd variables for each cluster.

\begin{tabular}{lccc}
\hline & Cluster 1 & Cluster 2 & Cluster 3 \\
\hline Number of herds & 403 & 207 & 140 \\
Proportion of herds: & & & \\
$\quad$ Australia & 32.6 & 53.0 & 14.4 \\
Canada & 77.7 & 12.3 & 9.9 \\
$\quad$ United States & 54.8 & 24.6 & 20.6 \\
$\quad$ South Africa & 64.3 & 7.1 & 28.6 \\
Herd size & & 107 & 46 \\
$\quad$ (number of first lactations) & 14 & 5740 & 5736 \\
305-d yield (kg) & 5527 & 932 & 976 \\
Herd SD (kg) & 907 & 84 & 69 \\
Days to milk peak yield & 59 & & \\
\hline
\end{tabular}

weather conditions for such production systems. The larger variation of contemporary group effects in $\mathrm{ZAF}$ can be partly attributed to the wide range of management systems in this country.

\section{HC}

Three clusters were identified (Table 5). The majority of herds from CAN, USA, and ZAF were in cluster 1; this cluster consisted of mainly small herds with relatively low within-herd variation of milk production, and early peak production. The majority of the AUS herds were in the second cluster, that also contained about one fourth of the USA herds. Herds in this cluster were small as well, but peak production was somewhat later in lactation, and more variation in milk production existed within these herds. The third cluster was made up by the somewhat larger herds from USA and ZAF with a relatively high level of within-herd variation.

Posterior mode of the heritability in cluster 1 and 3 was 0.36 and 0.34 , respectively, but was lower for cluster 2 (Table 6). Posterior mode for the phenotypic variance in cluster 3 was only slightly higher compared with cluster 2 , but a proportionately larger part of the variation in cluster 3 was genetic. The genetic correlations between the second and the other clusters were 0.91 and 0.92 (Table 6), indicating that the production environment in herds assigned to cluster 2 was slightly different from herds in the other clusters.

Table 6. Modes and SD (in subscript) of the posterior distribution of the sire and residual variances $\left(\times 1000 \mathrm{~kg}^{2}\right)$, heritabilities and genetic correlations for the multiple-trait herd cluster model.

\begin{tabular}{|c|c|c|c|}
\hline & Cluster 1 & Cluster 2 & Cluster 3 \\
\hline$\sigma_{s}^{2}$ & $\begin{array}{ll}64.6 \quad 8.2\end{array}$ & $63.8 \quad 12.2$ & $\begin{array}{lll}75.2 & 8.8\end{array}$ \\
\hline $\begin{array}{l}\sigma_{e}^{2} \\
\mathrm{~h}^{2} / \mathrm{r}_{\mathrm{g}}\end{array}$ & $\begin{array}{l}700.18 .7 \\
0.36^{0.039}\end{array}$ & $\begin{array}{l}787.8 \\
0.92 \quad 0.5 \\
0.29 \quad 0.054\end{array}$ & $\begin{array}{cc}790.5 & 8.6 \\
0.95 & 0.018 \\
0.91 & 0.035 \\
0.34 & 0.037\end{array}$ \\
\hline
\end{tabular}

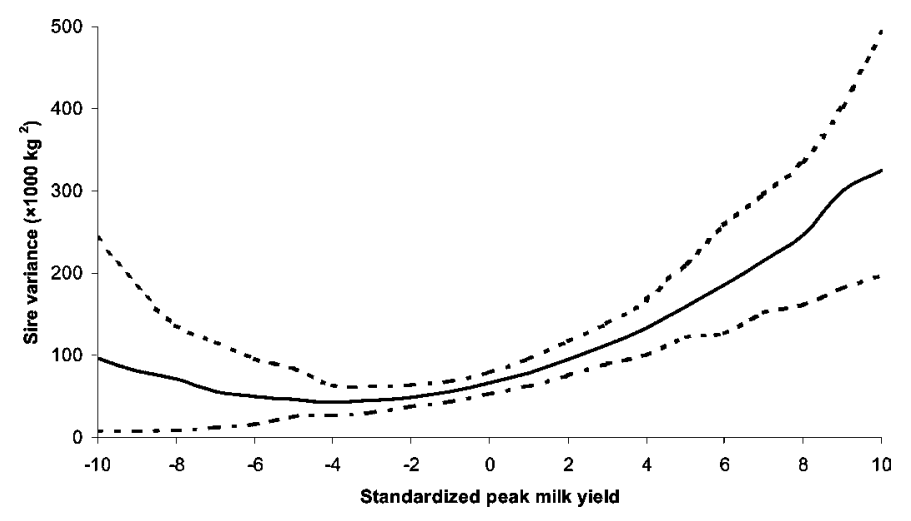

Figure 1. Posterior mode, and lower and higher bound of the 95\% highest posterior density region for sire variance of 305-d milk production as a function of standardized herd average for peak milk yield, for the reaction norm model.

Posterior SD of genetic correlations were much lower for model HC compared with model MT (Tables 4 and 6). This may be a consequence of the numerous genetic ties between clusters because many USA herds were present in all clusters. Genetic ties between countries, on the other hand, were created by commonly used AI bulls and were less strong. The distribution of observations was also more uniform over clusters than over countries, which probably explains why the range of posterior SD of variance components was smaller for model HC compared with models SThet and MT.

\section{RR}

Sire variances for the intercept and the slope of reaction norms for sires were 66,400 and $1360 \mathrm{~kg}^{2}$, respectively. The posterior mode for the genetic correlation between the intercept and the slope of reaction norms was 0.57 . The residual variance, with a value of 755,900 $\mathrm{kg}^{2}$, was close to that of the ST model (Table 3). The heritability in herds with an average level of milk peak yield was 0.33 , and the estimated correlation between milk production in extreme environments for milk peak yield was 0.62 .

The sire variance of 305-d milk production in each environment was computed using the sire variancecovariance matrix for the intercept and slope, as described by Kolmodin et al. (2002), and results hereof are in Figure 1. The sire variance of 305-d milk production increased with increasing herd average for milk peak yield over the major part of environment range. Standardized peak milk yield for $95 \%$ of the herds was between -5 and 4 , and in this interval the sire variance of 305 -d milk production increased from 47,000 to 134,000 $\mathrm{kg}^{2}$, comparable with results from literature (e.g., Zwald et al., 2003). An unusual pattern of sire variance 
Table 7. Results from the comparison between models. ${ }^{1}$

\begin{tabular}{lclr}
\hline & Deviance & $\begin{array}{l}\text { Eff. no } \\
\text { parameters }\end{array}$ & DIC \\
\hline ST & 0 & 6372 & 0 \\
SThet & -788 & 6411 & -749 \\
MT & -796 & 6433 & -734 \\
HC & -181 & 6464 & -89 \\
RR & -64 & 6491 & 77 \\
\hline
\end{tabular}

${ }^{1} \mathrm{ST}=$ single-trait model; SThet = single-trait model with heterogeneous residual variance across countries; $\mathrm{MT}=$ multiple-trait across country model; $\mathrm{HC}=$ multiple-trait herd cluster model; $\mathrm{RR}=$ reaction norm model (random regression).

at the extremes of the environment range was observed, but these sire variances were estimated with more uncertainty (Figure 1). In addition, the sire variance of 305-d milk production in each environment is a quadratic function of the value for the environmental descriptor (Kolmodin et al., 2002), and the few observations in the extreme environments had relatively little influence on the actual shape of the parabola. Other functions to describe reaction norms, in combination with transformation of the environmental descriptor, may be needed to improve the behavior of the reaction norm model at the extremes.

\section{Model Comparison}

Model MT was best regarding the goodness of fit to the data, followed by model SThet (Table 7). both evaluation models that ignore country borders (HC and RR) showed an improved goodness of fit compared with model ST, but not compared with the conventional analyses that acknowledges country borders (MT). Comparison of DIC values indicated that improved fit for some of the models was at the expense of an increased model complexity. Notably, the larger number of parameters in model MT compared to model SThet reversed the order of the models in favor of model SThet which had the lowest DIC value.

\section{DISCUSSION}

One of the advantages of using the $\mathrm{HC}$ model for international genetic evaluations is a reduction of the dimension of the genetic (co)variance matrix because the number of production environments is expected to be smaller than the number of countries. Lower dimension of (co)variance matrices means fewer genetic and phenotypic parameters are involved, and these can be estimated more precisely. In our study, the reduction was only from four countries to three production environments, but in similar studies with Holstein data the reduction of number of traits was over 50\% (Weigel and Rekaya, 2000; Zwald et al., 2001).
Genetic correlations between clusters were more precisely estimated than genetic correlations between countries (Tables 4 and 6) even though the difference in the order of the genetic (co)variance matrix between model HC and MT was only one. Surprisingly, both the goodness of fit and the effective number of parameters was in favor of model MT compared with model HC, suggesting that countries rather than production environments should be used for stratification of herds. The range of phenotypic variances was much larger for countries than for clusters, probably as a result of the presence of many USA herds in all three clusters (Table 5). Hence, phenotypic variances associated with AUS and ZAF records were probably too high with model $\mathrm{HC}$, causing the performance of this model to be below expectation.

The only difference between models RR and ST was the slope of the reaction norms for sires, i.e., the random linear regression of 305-d yield on the herd average for peak milk yield. The significant slope of the reaction norm found in this study implied that the genetic variance increased with higher peak milk yield in herds, i.e., more genetic variance existed in the better environments (Figure 1). The random regression model used in this study did not allow for heterogeneity of residual variances. However, results from models ST, SThet, and MT indicated that residual variances were heterogeneous. Relaxing this assumption may improve the goodness of fit and the comparison with the other models in this study. Other improvements worth considering are inclusion of additional environmental descriptors in the reaction norms and inclusion of linear regressions on environmental descriptors nested within fixed effects classes to allow effects to change over environments (e.g., the effect of age at calving may not be the same in herds with high and low peak milk yield).

The most basic categorization of genotype $\times$ environment interaction is to distinguish between scaling effect and reranking. The high genetic correlations between clusters indicate few rank changes of animals over clusters. In addition, genetic correlations among countries were estimated with much uncertainty, and values near unity were not unlikely. Thus, the presence of a genotype $\times$ environment interaction that causes reranking was not supported by grouping herds according to country or production environment. On the other hand, both $\mathrm{MT}$ and $\mathrm{HC}$ analysis showed that genetic and residual variances were heterogeneous over production environments. For the country analysis, the DIC value for model SThet was better than for model MT even though homogeneity of the genetic variance was assumed. Model MT was, however, handicapped by the lack of information to estimate genetic correlations among countries, whereas the assumption of homogeneous 
variance probably had relatively little impact due to the dominance of USA herds. A model that accounts for heterogeneous genetic and residual variances but with unity genetic correlation between performance in production environments may perform better than the models studied in the present investigation.

Explanatory effects were the same in all five models, whereas the models for national evaluation in the four countries differ considerably (Interbull, 2000). For example, the national evaluation model in USA contains an effect of registry status and a herd $\times$ sire interaction, season is not part of the contemporary group definition in ZAF, and AUS considers only two season per year. All models in this study can be modified to include country-specific explanatory effects, but variation in type and definition of explanatory effects should be kept at a minimum to avoid so-called genotype $\times$ model interactions.

National milk recording schemes in the four participating countries are organized in accordance with international standards, and unstandardized test-day observations could easily be combined into a joint genetic evaluation. Sprengel et al. (2001) conducted a binational evaluation for milking speed, and faced a situation in which the trait was recorded very differently in both countries. The multiple-trait across-country model is well suited for such a situation, however, adaptation of the other models to accommodate for differently recorded traits is less straightforward if the genetic correlation among the recorded traits deviates from unity.

The prime interest of computing international genetic evaluations is to predict performance of future offspring in any country or production environment. The criterion for model comparison used in this study summarized the goodness of fit of each model to the data. However, a better fit of the model to the data does not guarantee more accurate predictions of future records. Therefore, it is recommended to compare sire breeding values and rankings from each model, as well as to evaluate the ability of each model to predict future observations.

\section{CONCLUSIONS}

Models SThet and MT were better than the multipletrait herd cluster model and the reaction norm model for the Guernsey data based on the goodness of fit and model complexity. Residual and phenotypic variances were heterogeneous, which was most pronounced when herds were grouped according to country instead of production environments. There was little support from the data to treat performance in different production environments (countries) as separate traits, since values near unity for the genetic correlations were not unlikely given the high model and SD of the posterior densities, and because SThet had a better value for the deviance information criterion than model MT. We hypothesize that a model that allows for heterogeneous sire and residual variances will have a better fit than any of the models presented in this study.

\section{ACKNOWLEDGMENTS}

Financial support by the World Guernsey Federation, and the US Department of Agriculture/Emerging Market Program is gratefully acknowledged. We also want to thank ADHIS, Australia, CDN, Canada, USDAAIPL, USA and ARC, Republic of South Africa for providing the data used in this study.

\section{REFERENCES}

Fikse, W. F., R. Rekaya, and K. A. Weigel. 2003. Assessment of environmental descriptors for studying genotype by environment interaction. Livest. Prod. Sci. (accepted).

Interbull. 2000. National genetic evaluation programmes for dairy production traits practiced in Interbull member countries 19992000. Interbull Bull. 24.

Kinghorn, B. P. 1998. Mate selection by groups. J. Dairy Sci. 81:55-63.

Kolmodin, R., E. Strandberg, P. Madsen, J. Jensen, and H. Jorjani. 2002. Genotype by environment interaction in Nordic dairy cattle studied using reaction norms. Acta Agric. Scand. A Anim. Sci. $52: 11-24$.

NAG. 2000. NAG Fortran library manual. G03EFF: K-means cluster analysis. http://www.nag.co.uk/numeric/fl/manual/pdf/G03/ g03eff.pdf. Accessed August 3, 2000.

Pedersen, J., C. Langdahl, J. Pösö, and K. Johansson. 2001. A joint Nordic animal model for milk production traits in Holsteins and Ayrshires. Interbull Bull. 27:3-8.

Schmitz, F., R. W. Everett, and R. L. Quaas. 1991. Herd-year-season clustering. J. Dairy Sci. 74:629-636.

Spiegelhalter, D. J., N. G. Best, and B. P. Carlin. 1998. Bayesian deviance, the effective number of parameters and the complexity of arbitrary complex models. Technical report, Medical Research Council, Biostatistics Unit, Cambridge, UK.

VanKaam, J. B. C. H. M. 1998. Gibanal-Analyzing program for Markov Chain Monte Carlo sequences. Department of Animal Sciences, Wageningen Agricultural University.

Weigel, K. A., and R. Rekaya. 2000. A multiple-trait herd cluster model for international dairy sire evaluation. J. Dairy Sci. 83:815-821.

Weigel, K. A., R. Rekaya, N. R. Zwald, and W. F. Fikse. 2001. International genetic evaluation of dairy sires using a multiple-trait model with individual animal performance records. J. Dairy Sci. 84:2789-2795.

Zwald, N. R., K. A. Weigel, W. F. Fikse, and R. Rekaya. 2003. Identification of factors that cause genotype by environment interaction between herds of Holstein cattle in seventeen countries. J. Dairy Sci. 86:1009-1018.

Zwald, N. R., K. A. Weigel, and R. Rekaya. 2001. Application of a multiple-trait herd cluster model for genetic evaluation of dairy sires from seventeen countries. Interbull Bull. 27:13-18. 